\title{
Special Issue on the Sixteenth International Symposium on Robotics Research, 2013
}

\author{
The International Journal of \\ Robotics Research \\ 2015, Vol. 34(7) 835-836 \\ (C) The Author(s) 2015 \\ Reprints and permissions \\ sagepub.co.uk/journalsPermissions.nav \\ DOI: $10.1177 / 0278364915586155$ \\ ijr.sagepub.com
}

This special issue of The International Journal of Robotics Research (IJRR) contains extensions of papers presented at the 16th International Symposium on Robotics Research (ISRR), which took place in Singapore, 16-19 December 2013. ISRR is a biennial meeting organized by the International Federation of Robotics Research (IFRR); it has been running since 1983, making it one of the longestrunning robotics conferences.

There were 29 submitted and 16 invited papers presented at ISRR 2013. This special issue invited extensions of the ISRR conference papers to be submitted to IJRR, and these went through the full journal review process. The result is a collection of 10 fantastic papers on a wide variety of topics, representing exciting new directions in robotics. Instead of putting them in categories, we will briefly describe each in turn.

With computing resources on the rise and the price of cameras on the decline, we are seeing a lot more robotic systems using suites of many sensors to increase coverage and robustness. While multi-camera systems are becoming useful in such applications as self-driving cars and unmanned aerial vehicles, they also present new challenges when it comes to pose estimation. In Minimal solutions for the multi-camera pose estimation problem, Lee, Li, Pollefeys, and Fraundorfer address this issue head on by deriving new minimal solutions to estimating the position and orientation of calibrated multi-camera systems that are efficient and robust enough to use in real applications.

In the context of planning under uncertainty, the situation is different: irrespective of future growth of computational resources it will generally remain intractable to plan exactly in an uncertain world. Planning under uncertainty is most commonly formalized using partially observable Markov decision processes (POMDP). In their most general form they are intractable but good approximate solution methods exist. In the paper Planning in the continuous domain: A generalized belief space approach for autonomous navigation in unknown environments, the authors Indelman, Carlone, and Dellaert propose a novel algorithm that addresses shortcomings of existing methods; in particular, instead of planning in a discretized domain, it is able to plan in a continuous domain. The key to the proposed approach is a dual-layer architecture with an inner inference layer, which performs prediction, and an outer decisional layer, which determines the control strategy. The advantages of this method are demonstrated in the context of a mobile robot task that combines exploration with bounding uncertainty.

Sampling-based motion planning in continuous spaces has long been an exciting paradigm for planning, in part because it allows for differential constraints to be enforced in the resulting solution. Recently, an optimal version of sampling-based planning was presented, RRT* (Karaman and Frazzoli, IJRR 30(7): 846-894, 2011), and the quest for further improvements continues. In Fast marching tree: A fast marching sampling-based method for optimal motion planning in many dimensions, Janson, Schmerling, Clark, and Pavone present an optimal sampling-based planner that draws samples first and then uses 'lazy' dynamic programming to 'march' outwards from the start to the goal. Not only is FMT* $^{*}$ faster than previously published optimal sampling-based planners (particularly for high-dimensional problems), the paper is the first to provide theoretical bounds on the convergence rate to the optimum.

Manipulation remains one of the great challenges in robotics. When physical contact between the robot and the environment are involved, minute errors in state estimation can lead to vastly different outcomes of actions and, indeed, decide between failure and success. The paper Pose estimation for planar contact manipulation with manifold particle filters by Koval, Pollard, and Srinivasa proposes a novel particle-filter-based approach to state estimation during planar manipulation based on contact sensors. They propose Manifold Particle Filters as a novel way of estimating an object's position relative to the robot's hand based on the information provided by binary contact sensors. The provided real-world experiments demonstrate that the proposed approach is able to track an object's position during planar pushing, based on commercially available contact sensors.

Many conceivable applications will require the deployment of teams of ground robots. These robots generally must exchange information via wireless communication. In addition to the task of the team, maintaining connectivity in the presence of line-of-sight obstructions may be a challenge in itself. The paper Adaptive communication in 
multi-robot systems using directionality of signal strength by Gil, Kumar, Katabi, and Rus addresses this problem by augmenting the robot team with members whose role it is to maintain connectivity and bandwidth in accordance with the task's requirements. They propose an algorithm that takes into account the real-time directional signal strength to make control decisions for the routing robots. This realtime feedback leads to robust communication behavior by being able to respond quickly to variations in communication bandwidth and signal strength.

Interest in unmanned aerial vehicles is on the rise for a variety of applications, including aerial mapping, package delivery, and journalism. Flying high above obstacles has its own challenges outdoors, but maneuvering indoors in tight, cluttered spaces is potentially much harder. In Aggressive flight of fixed-wing and quadrotor aircraft in dense indoor environments, Bry, Richter, Bachrach, and Roy demonstrate aggressive flights for both quadrotors (up to $8 \mathrm{~m} / \mathrm{s}$ ) and fixed-wing aircraft (up to $11 \mathrm{~m} / \mathrm{s}$ ). To accomplish this, they present novel trajectory planning and state estimation techniques that account for the full dynamics of their vehicles. The methods are both efficient and robust, as demonstrated by the fact that the planned trajectories were flown autonomously using the state estimation methods in-the-loop during the experiments.

Legged robots hold the promise of making accessible to robots not only large parts of the natural environments but also many man-made environments, tailored to the legged locomotion of humans. These advantages must be substantial enough to justify the additional cost incurred by legged locomotion in terms of mechanical and control complexity. To demonstrate the significant advantages of legged locomotion in spite of the additional cost, the paper Towards versatile legged robots through active impedance control by Semini, Barasuol, Boaventura, Frigerio, Focchi, Caldwell, and Buchli demonstrates exciting new capabilities. The key to their approach is active impedance. Their quadruped platform HyQ, weighing $80 \mathrm{~kg}$, is able to perform a flying trot on asphalt with purely impedancecontrolled legs and no springs in its mechanical structure. In addition, they demonstrate the flying trot on an indoor treadmill, resonant hopping, and squat jump landings.

Robotic manipulation has revolutionized many areas of manufacturing in the last few decades, but is typically used in environments that have been engineered for a given task; object grasping is a particularly difficult subproblem that is often simplified through specialized end effector and/or object design. Generalizing this success to unknown, unstructured, cluttered environments remains an open research challenge. In Exploitation of environmental constraints in human and robotic grasping, Eppner, Deimel, Álvarez-Ruiz, Maertens, and Brock propose and test the hypothesis that the secret to successful grasping (in humans and therefore robots) is to exploit environmental constraints to effectively simplify the grasping problem; for example, humans will slide a piece of paper to the edge of a table rather than attempt to pick it up directly. Through a variety of experiments, they use this concept to demonstrate both improved grasping strategies for existing robot hand designs and novel flexible hands that better exploit environmental constraints in grasping general objects.

Arguably, robotic systems that safely navigate urban environments will appear in our cities in the not-too-distant future. Until then, some important challenges must be overcome. Detecting, tracking, and avoiding unpredictably moving obstacles, such as pedestrians, clearly is one of them. The paper Model-free detection and tracking of dynamic objects with $2 D$ lidar by Wang, Posner, and Newman describes a novel, model-free approach to jointly estimating sensor pose, background, and the dynamic states of moving obstacles. Taking a model-free approach enables the approach to address unexpected object classes, a necessity for deployment in urban environments. The effectiveness of the approach is demonstrated using realworld data.

Semantic scene perception and understanding remains a tremendous challenge, in particular in complex, dynamic, everyday environments. Sensor limitations, occlusions, and the robot's motion all make it necessary to merge information obtained from multiple viewpoints into a consistent scene representation. This robustness of this merging process critically depends on an accurate solution to the data association problem. The paper Data association for semantic world modeling from partial views by Wong, Kaebling, and Lozano-Pérez proposes several novel clustering-based data association approaches. These methods achieve impressive results in table-top scenes with many objects using only a fraction of the computation time of comparable data association approaches.

We hope you enjoy this special issue of IJRR. We would like to express our gratitude to the Co-chairs of ISRR, Masayuki Inaba and Peter Corke, the IFRR, the reviewers and authors, and finally John Hollerbach and Jennet Batten at IJRR for keeping us on track during the special issue editorial process.

Guest Editors:
Tim Barfoot
University of Toronto, Canada
tim.barfoot@utoronto.ca
Oliver Brock
che Universität Berlin, Germany
oliver.brock@tu-berlin.de

